\title{
DISSULFETO DE MOLIBDÊNIO, UM MATERIAL MULTIFUNCIONAL E SURPREENDENTE
}

\section{Fernando Wypych}

Departamento de Química, Universidade Federal do Paraná, CP 19081, 81531-990 Curitiba - PR

Recebido em 6/10/00; aceito em 4/4/01

\begin{abstract}
MOLYBDENUM DISULFIDE, A MULTIFUNCTIONAL AND REMARKABLE MATERIAL. The aim of this work is to review the chemical and physical properties of layered molybdenum disulfide. The three polymorphic/polytypic modifications of the compound were found, the polytypes $2 \mathrm{H}$ (molybdenite) and $3 \mathrm{R}$ are semiconductors while the polymorph $1 \mathrm{~T}$ is an electronic conductor. $2 \mathrm{H}-\mathrm{MoS}_{2}$ has several important industrial applications as hydrotreatment catalysts, energy storage devices, solar cells, solid lubricants, among others. When intercalated, the $2 \mathrm{H}$ phase changes to a distorted $1 \mathrm{~T}$ phase, producing unstable intercalation compounds that can be exfoliated in solution, producing single layers and consequently nanocomposites. The direct synthesis of the $1 \mathrm{~T}$ phase produces stable intercalation compounds. Recently molybdenum disulfide was prepared as nanotubes and fulerenelike structures that bring new insights in the investigation of this important material.
\end{abstract}

Keywords: molybdenum disulfide; intercalation; layered materials.

\section{INTRODUÇÃO}

O dissulfeto de molibdênio pertence a uma classe de materiais conhecidos como compostos lamelares e ocorre em três modificações polimórficas/politípicas ${ }^{1,2}$. A fase mais comum $(2 \mathrm{H})$ ocorre na natureza na forma de molibdenita. Nas modificações $2 \mathrm{H}$ e $3 \mathrm{R}$, átomos de molibdênio estão ligados covalentemente à átomos de enxofre numa geometria trigonal prismática regular (Figura 1). Os prismas trigonais estão ligados entre si pelas arestas, produzindo unidades bidimensionais conhecidas como lamelas. Essas lamelas que, estão conectadas por forças fracas do tipo Van der Waals, podem se ordenar de maneira diferente originando os dois politipos. Na fase $1 \mathrm{~T}$ a forma ondulada se deve à distorção da esfera de coordenação octaédrica do centro metálico ${ }^{2}$. A forma do politipo $1 \mathrm{~T}$ não distorcida está representada na Figura 1.

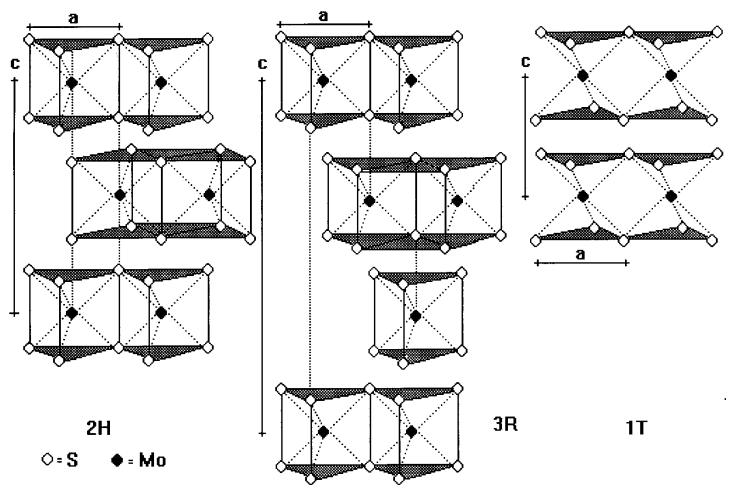

Figura 1. Representação esquemática das estrutura das fases $2 H$, $3 R$ e $1 T$ (não distorcida), do dissulfeto de molibdênio.

As propriedades dos politipos são diferentes. A fase $1 \mathrm{~T}$ é condutora $^{2}$ enquanto que as fases $2 \mathrm{H}$ e $3 \mathrm{R}$ são diamagnéticas e

\footnotetext{
*e-mail: wypych@quimica.ufpr.br
}

semicondutoras ${ }^{3}$. Além das três formas conhecidas de hábito cristalino, o dissulfeto de molibdênio se apresenta na forma amorfa ${ }^{4,5}$, como nanotubos $^{6,7}$ e esferas de dimensões nanoscópicas ${ }^{7,8}$ semelhantes as estruturas dos fulerenos.

As aplicações do dissulfeto de molibdênio vão desde baterias no estado sólido que utilizam meio não aquoso e íons lítio intercaláveis ${ }^{9-11}$, células solares ${ }^{12}$, como lubrificantes sólidos ${ }^{13}$, catalisadores ${ }^{14-18}$, onde desempenha um papel de importância fundamental, além de outras.

Um dos processos de síntese do dissulfeto de molibdênio nas fases polimórficas $2 \mathrm{H}$ e $3 \mathrm{R}$ é a reação térmica direta entre o molibdênio metálico e enxofre elementar em ampolas de quartzo. Quando cristais grandes forem necessários utiliza-se o método de transporte de vapor químico com um halogeneto como o iodo como agente transportante ${ }^{19}$ (Eq.1).

$\mathrm{Mo}+2 \mathrm{~S} \rightarrow \mathrm{MoS}_{2}+2 \mathrm{I}_{2} \leftrightarrow \mathrm{MoI}_{4}+\mathrm{S}_{2}$

Nesse procedimento, quantidades estequiométricas do metal na forma de pó e enxofre elementar, além de uma pequena quantidade de iodo metálico, são selados em uma ampola de quartzo evacuada. Outra possibilidade consiste em sintetizar o dissulfeto de molibdênio na forma de pó e selá-lo em uma ampola de quartzo na presença de iodo metálico. A mistura reacional é deslocada para a ponta da ampola, a qual é aquecida a uma determinada temperatura, posicionando a outra ampola em uma temperatura ligeiramente inferior. Tipicamente as dimensões da ampola são $3 \mathrm{~cm}$ de diâmetro e $20 \mathrm{~cm}$ de comprimento. A ponta da ampola com o material é aquecida à $950{ }^{\circ} \mathrm{C}$ e a outra à $900^{\circ} \mathrm{C}$, criando-se um gradiente de temperatura. Na parte mais quente da ampola o $\mathrm{MoS}_{2}$ reage com o iodo, formando-se $\mathrm{MoI}_{4}$ gasoso que migra para a parte mais fria. Nessas condições de temperatura, $\mathrm{o} \mathrm{MoI}_{4}$ reage novamente com o enxofre, depositando um cristalito de $\mathrm{MoS}_{2}$. Dependendo do tempo de reação, da quantidade de material, do diâmetro da ampola e quantidade de agente transportante, cristais de vários milímetros ou até centímetros podem ser obtidos (Eq. 1) ${ }^{19}$.

Outra metodologia menos comum envolve a decomposição do tetratiomolibdato de amônio sólido ${ }^{20-23}$ (Eq. 2). Nesse caso utilizan- 
do-se uma mistura de tetratiomolibdato e tetratiotungstato, soluções sólidas poderão potencialmente serem obtidas.

$$
\left(\mathrm{NH}_{4}\right)_{2} \mathrm{MoS}_{4} \rightarrow \mathrm{MoS}_{3}+2 \mathrm{NH}_{3}+\mathrm{H}_{2} \mathrm{~S} \rightarrow \mathrm{MoS}_{2}+1 / \mathrm{x} \mathrm{S}_{\mathrm{x}}
$$

Nesse caso existe uma certa divergência dos dados da literatura relacionada ao número de intermediários de reação porém é de consenso que intermediários amorfos são produzidos antes da cristalização do $\mathrm{MoS}_{2}$ final.

A sulfetação do óxido de molibdênio VI também é uma rota alternativa de síntese $\mathrm{e}^{4,24}$ (Eq.3), tendo sido recentemente descrita como adequada para a obtenção de dissulfeto de molibdênio com morfologias não usuais ${ }^{7}$. Nessa síntese, apesar do composto final ser o $2 \mathrm{H}-\mathrm{MoS}_{2}$, compostos intermediários ricos em enxofre $\left(\mathrm{MoS}_{3}\right)$ foram observados ${ }^{4}$.

$\mathrm{MoO}_{3}+2 \mathrm{H}_{2} \mathrm{~S} \rightarrow \mathrm{MoS}_{2}+2 \mathrm{H}_{2} \mathrm{O}+1 / 2 \mathrm{O}_{2}$

A precipitação de sulfetos amorfos em atmosfera inerte, seguido de posterior tratamento térmico conduz também ao $\mathrm{MoS}_{2}{ }^{5,9}$ (Eq.4).

$\mathrm{MoCl}_{4}+2 \mathrm{Li}_{2} \mathrm{~S} \rightarrow \mathrm{MoS}_{2}+4 \mathrm{LiCl}$

Outras metodologias menos usuais reportam a precipitação de sulfetos em meio ácido a partir de soluções de tiomolibdato de amônio $^{25}$ (Eq. 5) ou obtenção através de sulfetação de soluções ácidas de molibdatos (Eq. 5,6) ${ }^{25}$, ou ainda através de precursores orgânicos, utilizando-se fontes de energia como ultrasom ${ }^{26}$ ou plasma de microondas ${ }^{27}$.

$\left(\mathrm{NH}_{4}\right)_{2} \mathrm{MoS}_{4}+2 \mathrm{HCl} \rightarrow \mathrm{MoS}_{2}+2 \mathrm{NH}_{4} \mathrm{Cl}+\mathrm{H}_{2} \mathrm{~S}+1 / 2 \mathrm{~S}_{2}$

$\mathrm{K}_{2} \mathrm{MoO}_{4}+4 \mathrm{H}_{2} \mathrm{~S} \rightarrow \mathrm{K}_{2} \mathrm{MoS}_{4}+4 \mathrm{H}_{2} \mathrm{O}$

\section{USO EM CATÁLISE}

Desde a década de 50 tem se procurado por catalisadores mais eficientes para promover a remoção de contaminantes de combustíveis e consequentemente atender a legislação cada vez mais exigente no que tange às emissões veiculares. Um composto chave nessa busca envolve o dissulfeto de molibdênio dopado com níquel ou cobalto e ancorado na superfície de suportes inertes ${ }^{14-17}$ ou ainda altamente disperso no meio reacional ${ }^{18,28-30}$. O processo envolve a remoção de poluentes de combustíveis, na presença de hidrogênio.

Os poluentes mais comuns encontrados nos combustíveis na forma de heteroátomos envolvem o nitrogênio, oxigênio, enxofre e metais. Os processos de remoção dessas espécies origina as técnica conhecidas como hidrodenitrogenação (HDN), hidrodeoxigenação (HDO), hidrodesulfurização (HDS) e hidrodemetalização (HDM), respectivamente. Como durante o processo de tratamento catalítico ocorrem os vários processos simultaneamente, a técnica é genericamente conhecida como hidrotratamento (HDT). Devido à importância desse sistema e apesar das centenas de publicações anuais sobre o assunto com a existência de grupos de pesquisa que se dedicam quase que exclusivamente ao estudo em questão, não se desvendou claramente o mecanismo da atuação do dissulfeto de molibdênio e dopantes nos processos catalíticos.

Um dos problemas principais se situa no posicionamento do dopante na estrutura do catalisador, tendo sido praticamente excluído o efeito do suporte e efeito de compostos livres de molibdênio. Apesar dessa exclusão, o posicionamento do níquel (ou cobalto) na estrutura do dissulfeto é ainda um desafio que motiva estudos intensivos nessa área ${ }^{14-18,31-37}$. Algumas possibilidades foram aventadas (Figura 2), entre elas a possibilidade da intercalação do níquel no espaçamentos interlamelares internos (Figura 2-1), pseudo-intercalação nos espaçamentos interlamelares laterais (Figura 2-2), substituição dos átomos de molibdênio intralamelares (Figura 2-3), além da reação do níquel com a superfícies (Figura 2-4) ou laterais das lamelas (Figura 2-5).

A Figura 2 apresenta todas as possibilidades do posicionamento dos átomos de níquel na estrutura do dissulfeto de molibdênio (adaptado da ref. 14).

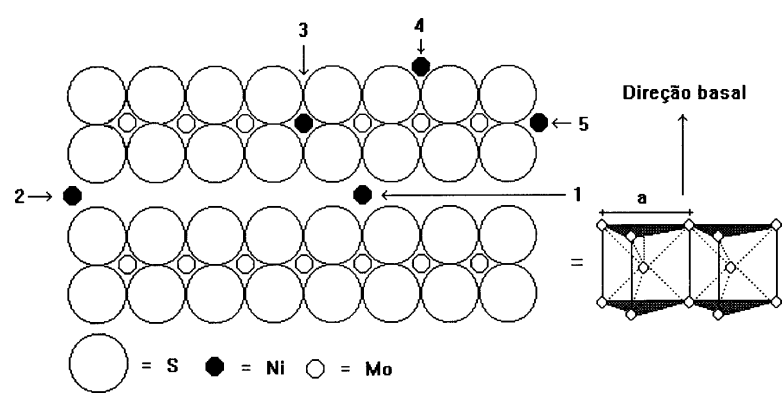

Figura 2. Possibilidades do posicionamento dos átomos de níquel na estrutura do dissulfeto de molibdênio.

Resultados recentes ${ }^{35}$ tem demonstrado que a fase ativa nesses catalisadores é gerada quando o dopante se coordena às laterais das lamelas produzindo uma fase do tipo Ni-Mo-S ${ }^{35}$ (Figura 2 - 5). Como a fase Ni-Mo-S é gerada somente nas laterais dos cristais, é de difícil caracterização provocando ainda divergências entre os pesquisadores.

Existem várias metodologias de síntese desses catalisadores porém a mais difundida envolve a impregnação de um suporte inerte com uma mistura de heptamolibdato de amônio e nitrato de níquel, seguido de processos de calcinação e sulfetação ${ }^{15,35}$. Os métodos mais recentes envolvem a complexação de molibdênio e níquel com agentes quelantes como ácido etilenodiaminotetracético (EDTA) ou ácido nitrilotriacético (NTA) seguido de impregnação e sulfetação ${ }^{35,37}$. Não é de nosso conhecimento que compostos mistos de molibdênio e níquel tenham sido sulfetados. Esse procedimento poderia gerar materiais interessantes pela mistura íntima dos dois metais que poderia preferencialmente produzir a fase ativa e não simplesmente "decorar" cristais de $\mathrm{MoS}_{2}$ com níquel.

Os suportes mais utilizados são alumina, sílica, carbono entre outros.

No caso da síntese de catalisadores dispersados no meio reacional ${ }^{28-30}$, utiliza-se a decomposição do tetratiomolibdato de amônio na presença de ácido sulfídrico e atmosfera redutora de hidrogênio $^{28-30}$ (eq. 2,7), sulfetação de heptamolibdato de amônio com ácido sulfídrico ${ }^{29}$ ou reação do tiocianato de potássio com óxido de molibdênio $^{30}$ (eq. 8).

$$
\begin{aligned}
& \left(\mathrm{NH}_{4}\right)_{2} \mathrm{MoS}_{4} \rightarrow \mathrm{MoS}_{3}+\left(\mathrm{NH}_{4}\right)_{2} \mathrm{~S} \rightarrow \mathrm{MoS}_{2}+1 / \mathrm{x} \mathrm{S}_{\mathrm{x}} \\
& \mathrm{MoO}_{3}+2 \mathrm{KSCN} \rightarrow \mathrm{MoS}_{2}+2 \mathrm{KOCN}+1 / 2 \mathrm{O}_{2}
\end{aligned}
$$

\section{USO EM LUBRIFICANTES SÓLIDOS}

A utilização das propriedades tribológicas desses sistemas está fundamentada basicamente na sua estrutura lamelar, nas suas boas propriedades anti-friccionais, boa adesão e estabilidade térmica e química. Esses materiais atuam em condições extremas de aqueci- 
mento ou resfriamento, onde lubrificantes líquidos comuns não seriam adequados ${ }^{13,38}$.

Existe um grande potencial na utilização de materiais intercalados com cátions volumosos devido a possibilidade do controle da interação interlamelar. Já que quando maior o afastamento das lamelas, menor o seu grau de interação, seria possível a utilização de íons esféricos ou camadas de solvatação desses íons, como nanorolamentos. Infelizmente sistemas desse tipo são bastante instáveis já que são intercalados normalmente com moléculas orgânicas, portanto passíveis de serem retiradas com facilidade, além da instabilidade do sistema $\mathrm{Mo}^{+4} / \mathrm{Mo}^{+3}$ nos compostos de intercalação resultantes. Nesse caso, um sistema mais promissor envolveria o uso do dissulfeto de nióbio como matriz de intercalação. A Figura 3 apresenta um protótipo ideal de tal sistema em funcionamento, onde a setas indicam o escorregamento das lamelas umas sobre as outras em cima dos nanorolamentos (esferas escuras preenchidas).

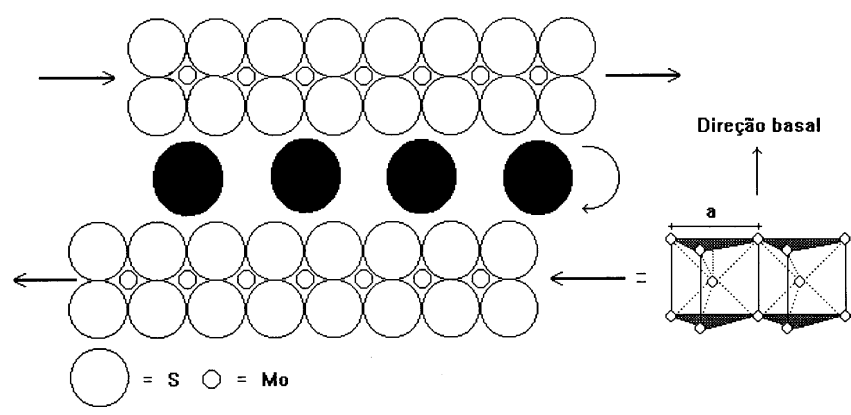

Figura 3. Protótipo de utilização de compostos de intercalação como lubrificantes sólidos.

\section{USO EM BATERIAS NO ESTADO SÓLIDO}

$2 \mathrm{H}-\mathrm{MoS}_{2}$ pode ser reduzido/oxidado em condições não aquosas na presença de íons lítio, o que proporciona um ciclo de descarregamento/ carregamento, típico de uma bateria no estado sólido (Eq. 9) 99-41. $^{39}$.

$\mathrm{MoS}_{2}+\mathrm{x} \mathrm{e}^{-}+\mathrm{x} \mathrm{Li}^{+} \leftrightarrow \mathrm{Li}_{x} \mathrm{MoS}_{2}$

Esse procedimento parece bastante simples já que os íons lítio de dimensões reduzidas (cerca de $0,5 \AA$ ) poderiam ser inseridos nos espaçamentos interlamelares e removidos facilmente provocando somente uma pequena expansão da rede cristalina, evitando desta forma uma expansão do eletrodo e ruptura da bateria. Existe um pequeno inconveniente que é a transição da fase estrutural que ocorre durante o processo de intercalação dos íons lítio. A coordenação trigonal prismática do molibdênio passa a octaédrica distorcida ${ }^{40-44}$. Essa transição de fase estrutural proporciona uma ruptura de ligações, o que danifica o cristal durante o processo de carga/descarga e consequentemente limitando a vida útil da bateria. Apesar desse inconveniente, existem no mercado baterias baseadas no sistema exposto acima ${ }^{45}$. Processos de intercalação derivados do $1 \mathrm{~T}-\mathrm{MoS}_{2}$ serão descritos abaixo.

\section{NANOCOMPÓSITOS}

Os processos de intercalação de moléculas de grandes dimensões nos espaçamentos interplanares dos sistemas lamelares são impedidos cineticamente principalmente pela baixa difusão destas. Para contornar esse inconveniente desenvolveu-se uma técnica de esfoliação (separação das lamelas individuais) utilizando-se métodos químicos. O procedimento consiste em intercalar lítio $(\mathrm{x} \approx 1)$ nos espaçamentos interplanares através do uso de uma solução de n- butillítio (n-BuLi) em hexano e atmosfera inerte e posteriormente utilizar a instabilidade do sistemas em meio aquoso, para processar a oxidação das lamelas e redução do lítio como descrito nas equações 10 e 11. A reação de produção de hidrogênio entre as lamelas, com o auxílio de um banho de ultra-som, produz a suspensão de monolamelas para uso posterior.

$$
\begin{gathered}
\mathrm{MoS}_{2}+\mathrm{x} \mathrm{n}-\mathrm{BuLi} \rightarrow \mathrm{Li}_{x} \mathrm{MoS}_{2}+\mathrm{x} / 2 \text { n-octano } \\
\mathrm{Li} \mathrm{MoS}_{2}+\mathrm{y}+\mathrm{z} \mathrm{H}_{2} \mathrm{O} \rightarrow \mathrm{Li}_{x-\mathrm{y}}\left(\mathrm{H}_{2} \mathrm{O}\right)_{\mathrm{z}} \mathrm{MoS}_{2}(\text { mon. })+ \\
+\mathrm{y} \mathrm{LiOH}+\mathrm{y} / 2 \mathrm{H}_{2}
\end{gathered}
$$

As monolamelas obtidas, após lavagem de LiOH obtido, ainda apresentam uma certa quantidade de íons lítio hidratados presos às lamelas $(x-y \approx 0,1)$, podem ser utilizadas para a intercalação de outras moléculas não diretamente intercaláveis, como os polímeros condutores iônicos/eletrônicos (eq. 12) (6-64 $^{\text {. }}$

$\mathrm{Li}_{x-y}\left(\mathrm{H}_{2} \mathrm{O}\right) \mathrm{MoS}_{2}\left(\right.$ mon.) + w polímero $\rightarrow \mathrm{Li}_{x-y}\left(\mathrm{H}_{2} \mathrm{O}\right)_{\mathrm{z}-\mathrm{w}}$
(polímero) $\mathrm{MoS}_{2}+\mathrm{w} \mathrm{H}_{2} \mathrm{O}$

Não existe um consenso na literatura sobre o mecanismo dessa síntese porém reconhece-se que existe uma certa percentagem de lítio na amostra, além do polímero e água.

Os materiais obtidos apresentam propriedades interessantes que normalmente são originadas do sinergismo das propriedades da matriz original e molécula intercalada. Com o intuito de se produzir materiais especiais, tem sido despendidos esforços na preparação desses materiais nanocompósitos com polímeros.

Através do método de esfoliação, além dos polímeros, uma série de compostos não diretamente intercaláveis, foram intercalados no dissulfeto de molibdênio derivado da fase $2 \mathrm{H}^{53-65}$.

\section{COMPOSTOS DE INTERCALAÇÃO}

O estudo dos compostos de intercalação derivados do dissulfeto de molibdênio foram estimulados pela obtenção de compostos com propriedades metálicas e até supercondutoras à baixas temperaturas $(\sim 4-7 \mathrm{~K})^{3}$. Os estudos em sistemas lamelares baseados no sulfetos metálicos motivaram a descoberta dos supercondutores baseados em sistemas do tipo óxidos mistos lamelares no final da década de 80 .

Os primeiros experimentos de intercalação do $2 \mathrm{H}-\mathrm{MoS}_{2}$ utilizavam soluções de metais alcalinos e alcalino terrosos dissolvidos em amônia líquida ou exposição do $\mathrm{MoS}_{2}$ a vapores do metal alcalino, à quente. As fases ternárias obtidas se mostraram bastante instáveis, regenerando a fase original, após exposição do composto de intercalação ao ar úmido. Sob atmosfera controlada puderam ser isolados compostos de intercalação, porém suas caracterizações estruturais nunca foram efetivamente realizadas, dificultadas principalmente pela baixa qualidade dos cristais após o processo de intercalação.

Uma das metodologias descritas mais recentemente envolvia o uso de n-butillítio como reagente redutor ${ }^{43,46-51,54,56-58}$ (Eq.10). Nesse caso, os compostos também se apresentavam com desordem estrutural porém algumas caracterizações e a transição de fase $2 \mathrm{H} \rightarrow 1 \mathrm{~T}$ foram propostas ${ }^{40-43}$. Mais recentemente foi sintetizada a fase metaestável 1T, reconhecida como a terceira fase polimórfica do dissulfeto de molibdênio ${ }^{2}$. Essa fase é obtida em três passos distintos de síntese. O primeiro consiste na sulfetação do molibdato de potássio (Eq. 6).

Em seguida o composto obtido é reduzido com uma mistura de nitrogênio/hidrogênio à temperatura elevada (Eq. 13).

$2 \mathrm{~K}_{2} \mathrm{MoS}_{4}+3 \mathrm{H}_{2} \rightarrow 2 \mathrm{~K}_{1-\mathrm{x}} \mathrm{MoS}_{2}+2 \mathrm{x} \mathrm{K}^{0}+\mathrm{K}_{2} \mathrm{~S}+3 \mathrm{H}_{2} \mathrm{~S}$ 
A fase obtida é altamente higroscópica e pode ser parcialmente oxidada, ajustando-se o $\mathrm{pH}$ da solução de lavagem próximo a neutralidade como descrito na Eq. 14.

$\mathrm{K}_{1-\mathrm{x}} \mathrm{MoS}_{2}+\mathrm{y}+\mathrm{z} \mathrm{H} \mathrm{H}_{2} \mathrm{O} \rightarrow \mathrm{K}_{1-\mathrm{x}-\mathrm{y}}\left(\mathrm{H}_{2} \mathrm{O}\right)_{\mathrm{z}} \mathrm{MoS}_{2}+\mathrm{y} \mathrm{KOH}+\mathrm{y} / 2 \mathrm{H}_{2}$

A fase $1 \mathrm{~T}-\mathrm{MoS}_{2}$ é obtida através da oxidação total da fase ternária com um oxidante de potencial adequado (Eq. 15) (Iodo em acetonitrila ou dicromato de potássio em solução diluída de ácido sulfúrico).

$\mathrm{K}_{1-\mathrm{x}-\mathrm{y}}\left(\mathrm{H}_{2} \mathrm{O}\right) \mathrm{MoS}_{2}+$ oxidante $\rightarrow 1 \mathrm{~T}-\mathrm{MoS}_{2}+1-\mathrm{x}-\mathrm{y} \mathrm{K}$

Ao contrário das fases $2 \mathrm{H}$ e $3 \mathrm{R}$, a fase $1 \mathrm{~T}$ apresenta uma estrutura característica derivada do composto ternário intercalado com potássio, onde os átomos de molibdênio ocupam sítios octaédricos distorcidos, conforme comprovado por várias técnicas ${ }^{66-68}$. A fase é muito semelhante àquela obtida através do processo de intercalação de $\mathrm{n}-\mathrm{BuLi}$ na fase $2 \mathrm{H}$, porém a última é comparativamente muito mais estável e cristalina.

Os compostos de intercalação derivados da fase $1 \mathrm{~T}-\mathrm{MoS}_{2}$ ou do composto $\mathrm{K}_{\mathrm{x}}\left(\mathrm{H}_{2} \mathrm{O}\right)_{\mathrm{y}} \mathrm{MoS}_{2}$ são mais estáveis e podem gerar por troca iônica ou substituição de solventes vários derivados, como descrito anteriormente ${ }^{66-73}$. Reações de troca iônica (Eq. 16) conduzem à compostos de intercalação monoclínicos (Tabela 1).

$\mathrm{K}_{\mathrm{x}}\left(\mathrm{H}_{2} \mathrm{O}\right)_{\mathrm{y}} \mathrm{MoS}_{2}+\mathrm{y} \mathrm{A}^{+\mathrm{n}} \rightarrow \mathrm{A}_{\mathrm{y} / \mathrm{n}}\left(\mathrm{H}_{2} \mathrm{O}\right)_{\mathrm{z}} \mathrm{MoS}_{2}+\mathrm{x} \mathrm{K}$

Existia uma controvérsia na literatura relacionada com a superestrutura obtida nos derivados de intercalação da fase $2 \mathrm{H}$ e $1 \mathrm{~T}^{40-}$ $42,65,66-74$ porém a estrutura reportada anteriormente ${ }^{73}$ foi recentemente confirmada ${ }^{66-68}$, sendo atualmente aceita como correta ${ }^{74,75}$.

A fase $\mathrm{K}_{\mathrm{x}} \mathrm{MoS}_{2}$ possui uma estrutura triclínica com uma superestrutura do tipo $\sim 2 \mathrm{a} x \sim 2 \mathrm{a}$ ( $\mathrm{a}=$ parâmetro de rede "a" = "b" do $2 \mathrm{H}$ $\mathrm{MoS}_{2}=3,31 \AA$ ), a fase $\mathrm{K}_{\mathrm{x}}\left(\mathrm{H}_{2} \mathrm{O}\right) \mathrm{yoS}_{2}$ possui uma estrutura monoclínica do tipo $\sim \mathrm{a} x \sim \mathrm{a} \sqrt{3}$ e a fase totalmente oxidada possui uma estrutura hexagonal do tipo $\sim a \sqrt{ } 3 \mathrm{x} \sim a \sqrt{ } 3^{66-68,74,75}$.

Os parâmetros de rede basais dos compostos de intercalação derivados do $1 \mathrm{~T}-\mathrm{MoS}_{2}$ versus a razão carga/raio (q/r) do cátion intercalado pode ser observados nas figuras 4 (eixo c), 5 (eixo a) e 6 $(\text { eixo b })^{73}$.

Observa-se na Figura 4, três grupos de parâmetro de rede, o primeiro nos cátions com razão carga raio até 1 que possuem um parâmetro de rede médio de $9,41 \AA$. O segundo grupo com razão carga/raio entre 1 e 3, com um parâmetro de rede médio de 12,19 ̊ e

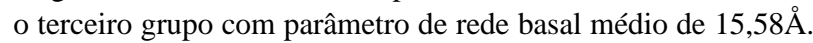

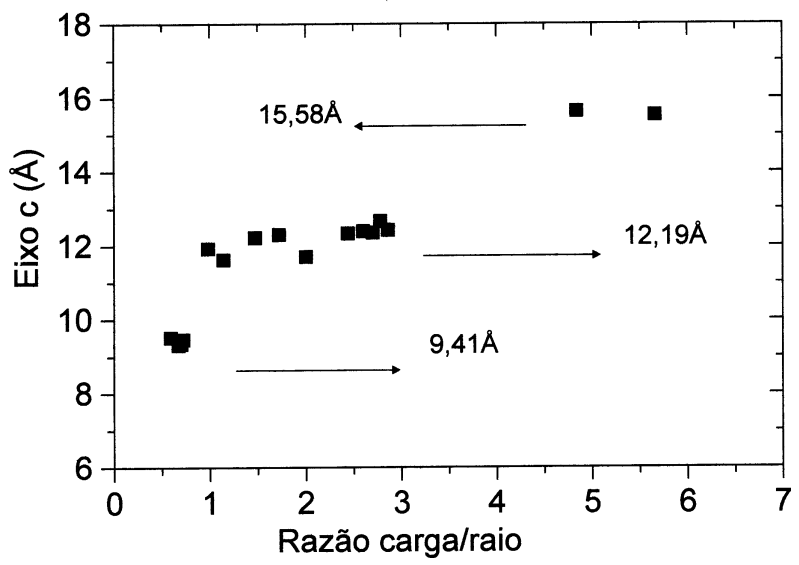

Figura 4. Parâmetro de rede basal versus razão carga/raio do cátion intercalado.

As variações de parâmetro de rede basais entre a matriz vazia $(5,99 \AA)$ e o grupo de $9,41 \AA$ é de $3,42 \AA$, entre o grupo de $12,19 \AA$ é de $6,2 \AA$ e entre o grupo de $15,58 \AA$ é de $9,56 \AA$. Os valores das variações dos espaçamentos são próximos de múltiplos de $3 \AA$, que é o diâmetro nominal da molécula de água. $(3,42 / 1=3,42 \AA ; 0,2 / 2=$ $3,1 \AA$ e $9,56 / 3=3,2 \AA$ ). Os grupos correspondem à intercalação de

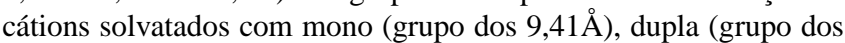
$12,19 \AA$ ) e tripla camadas de moléculas de águas de hidratação (grupo de $15,58 \AA$ ). Exclui-se nesses cálculos as possíveis interações entre as moléculas de água intercaladas e entre as lamelas e diferentes distorções das lamelas entre as fases intercaladas e vazia. Essas tendências foram observadas anteriormente para a intercalação de cátions inorgânicos hidratados em $2 \mathrm{H}-\mathrm{NbS}_{2}{ }^{76}$.

Observa-se que os parâmetros de rede não basais experimentam uma pequena variação, não sistemática, em função do cátion intercalado. Obviamente que durante o processo de intercalação, distorções significativas das lamelas não são possíveis principalmente baseado no fato de que todas as reações foram conduzidas à temperatura ambiente porém pequenas distorções podem estar presentes. Essas pequenas distorções poderiam eventualmente ser detectadas por difração de raios-X em monocristais entretanto são raros os casos em que monocristais de qualidade podem ser obtidos em sistemas lamelares. Considerando-se que as distorções são muito pequenas considerou-se

Tabela 1. Parâmetros de rede dos derivados de intercalação obtidos através de reações de troca iônica.

\begin{tabular}{|c|c|c|c|c|c|}
\hline Cátion & a $(\AA$ & $\mathrm{b}(\AA)$ & $c(\AA)$ & $\beta\left({ }^{\circ}\right)$ & $\mathrm{q} / \mathrm{r}$ \\
\hline $\mathrm{Li}^{+}$ & $5,704 \pm 0,003$ & $3,254 \pm 0,002$ & $11,617 \pm 0,005$ & $96,73 \pm 0,04$ & 1,14 \\
\hline $\mathrm{Na}^{+}$ & $5,713 \pm 0,002$ & $3,263 \pm 0,001$ & $11,927 \pm 0,005$ & $97,08 \pm 0,05$ & 0,98 \\
\hline $\mathrm{K}^{+}$ & $5,700 \pm 0,001$ & $3,238 \pm 0,001$ & $9,460 \pm 0,002$ & $100,43 \pm 0,02$ & 0,72 \\
\hline $\mathrm{Rb}^{+}$ & $5,710 \pm 0,002$ & $3,263 \pm 0,001$ & $9,310 \pm 0,003$ & $100,54 \pm 0,03$ & 0,67 \\
\hline $\mathrm{Cs}^{+}$ & $5,707 \pm 0,003$ & $3,250 \pm 0,002$ & $9,518 \pm 0,005$ & $99,05 \pm 0,06$ & 0,59 \\
\hline $\mathrm{Mg}^{+2}$ & $5,702 \pm 0,003$ & $3,275 \pm 0,003$ & $12,665 \pm 0,008$ & $97,51 \pm 0,06$ & 2,78 \\
\hline $\mathrm{Ca}^{+2}$ & $5,710 \pm 0,003$ & $3,257 \pm 0,001$ & $11,702 \pm 0,004$ & $95,93 \pm 0,04$ & 2,00 \\
\hline $\mathrm{Sr}^{+2}$ & $5,705 \pm 0,004$ & $3,270 \pm 0,003$ & $12,297 \pm 0,008$ & $95,25 \pm 0,07$ & 1,72 \\
\hline $\mathrm{Ba}^{+2}$ & $5,703 \pm 0,009$ & $3,240 \pm 0,006$ & $12,215 \pm 0,03$ & $97,25 \pm 0,20$ & 1,47 \\
\hline $\mathrm{Cr}^{+3}$ & $5,686 \pm 0,003$ & $3,241 \pm 0,004$ & $15,637 \pm 0,01$ & $95,73 \pm 0,07$ & 4,84 \\
\hline $\mathrm{NH}_{4}^{+}$ & $5,697 \pm 0,003$ & $3,246 \pm 0,002$ & $9,343 \pm 0,004$ & $100,45 \pm 0,05$ & 0,70 \\
\hline $\mathrm{Al}^{+3^{4}}$ & $5,700 \pm 0,001$ & $3,228 \pm 0,003$ & $15,518 \pm 0,008$ & $94,34 \pm 0,06$ & 5,66 \\
\hline $\mathrm{Fe}^{+2}$ & $5,702 \pm 0,003$ & $3,232 \pm 0,001$ & $12,388 \pm 0,008$ & $96,77 \pm 0,08$ & 2,60 \\
\hline $\mathrm{Co}+2$ & - & - & 12,35 & - & 2,70 \\
\hline $\mathrm{Ni}+2$ & - & - & 12,42 & - & 2,86 \\
\hline
\end{tabular}


as lamelas rígidas durante o processo de intercalação o que permite por aproximação, os cálculos acima citados (Figuras 5 e 6).

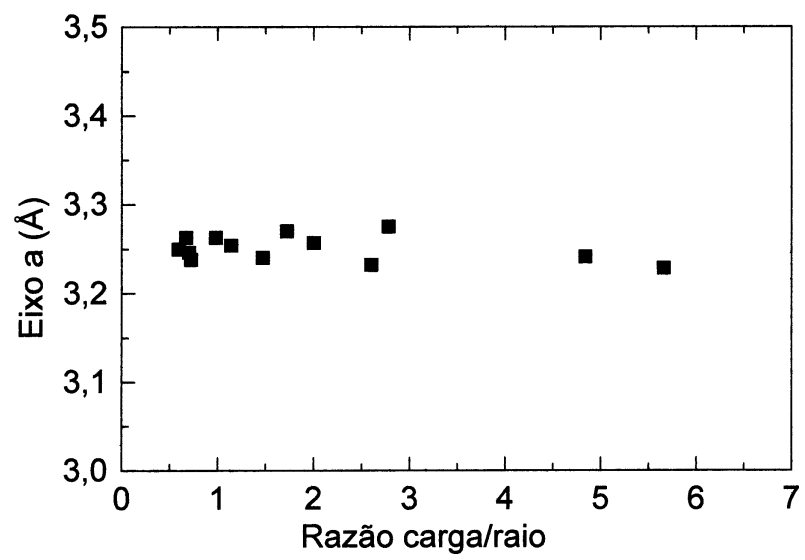

Figura 5. Parâmetro de rede "a" versus razão carga/raio do cátion intercalado.

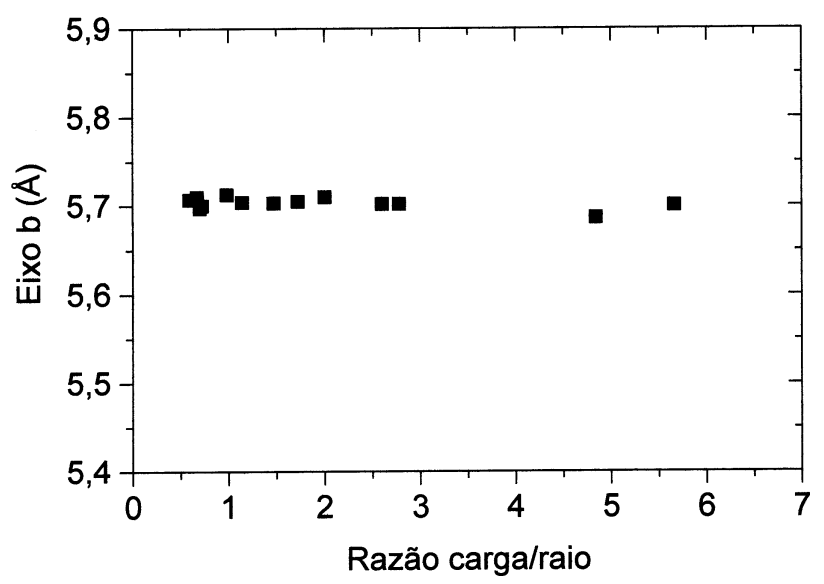

Figura 6. Parâmetro de rede " $b$ " versus razão carga/raio do cátion intercalado.

\section{OUTRAS ESTRUTURAS}

Além da obtenção do dissulfeto de molibdênio na forma de filmes finos orientados sobre vários substratos, recentemente observou-se que estes pode ser sintetizados na forma de nanotubos ${ }^{6}$ e estruturas conhecidas como materiais inorgânicos semelhantes aos fulerenos ${ }^{7,8}$. Esses materiais apresentam as mesmas lamelas constituintes nos materiais bidimensionais porém com uma expansão das distâncias interplanares basais de cerca de $2 \%(6,2 \AA)$ em relação ao politipo $2 \mathrm{H}$ características observadas $(6,15 \AA)$. Como as estruturas esféricas são multilamelares, a expansão foi atribuída à tensão gerada na dobradura das lamelas e diminuição dos tamanhos das lamelas internas.

Os processos de sínteses desses materiais envolvem desde o tradicional método de transporte químico até a reação do $\mathrm{MoO}_{3}$ com $\mathrm{H}_{2} \mathrm{~S}$ em uma atmosfera redutora de $\mathrm{H}_{2}$.

Além do crescimento de nanotubos e esferas, outros tipos de morfologias foram recentemente reportadas ${ }^{5}$.

\section{OUTRAS APLICAÇÕES}

Considerando-se que o $2 \mathrm{H}-\mathrm{MoS}_{2}$ é um semicondutor, o par elétron-buraco gerado a partir da irradiação com radiação eletromagné- tica de comprimento de onda adequado poderá ser utilizado em sistemas de conversão de luz em energia elétrica por células solares ${ }^{77,78}$ ou converter a energia luminosa em energia química ${ }^{79}$. Essa última poderá catalisar reações de fotólise da água ou fotodegradação de moléculas contaminantes de águas, procedimento que poderá ser adotado em processos de remediação de efluentes. Processos semelhantes são utilizados como semicondutores tradicionais como $\mathrm{TiO}_{2}$ e $\mathrm{ZnO}{ }^{80,81}$. Recentemente reportou-se a síntese ${ }^{26,79}$ e utilização de nanopartículas de dissulfeto de molibdênio na fotooxidação de moléculas orgânicas ${ }^{79}$. Observou-se que através do efeito quântico, após a diminuição do tamanho das partículas, até a luz solar poderia ser utilizada para realizar o processo de oxidação das moléculas orgânicas. $\mathrm{O}$ efeito quântico relacionado ao tamanho de partículas, alarga o "band gap" do semicondutor ao diminuir o tamanho de partícula, poderá ser utilizado de maneira vantajosa na preparação de nanoclusters de semicondutores de tamanhos variados, especialmente aqueles derivados de sulfetos metálicos como o $2 \mathrm{H}-\mathrm{MoS}_{2}$. Essa é uma tendência discutida na literatura recente.

\section{CONCLUSÕES}

O sulfeto de molibdênio é umas das substâncias mais versáteis que se conhece. Além das notável estabilidade química e térmica, pode ser crescido na forma de filmes finos, os quais possuem propriedades físicas anisotrópicas, o que a habilita para aplicações industriais variadas.

$\mathrm{O}$ politipo $2 \mathrm{H}$ possui ampla aplicação na indústria petrolífera especialmente importante como catalisador em sistemas de hidroconversão de combustíveis líquidos. Apesar da ausência de estudos catalíticos com a fase $1 \mathrm{~T}$, estudos preliminares na fase $2 \mathrm{H}$ esfoliada e adsorvida sobre alumina demonstrou um grande potencial em reações de metanação ${ }^{82}$. Essa constatação não é uma surpresa já que a área do catalisador e dispersão no substrato desempenham um papel fundamental na atividade desse. No caso da esfoliação do dissulfeto de molibdênio, monolamelas de dimensões reduzidas tendem ao caso ideal de dispersão num substrato.

As propriedades semicondutoras da fase $2 \mathrm{H}$ e possibilidades de produção desse material na forma de filmes finos, o torna um material com características ideais para a confecção de células solares conversoras de luz em energia elétrica.

$\mathrm{O}$ cristal da fase $2 \mathrm{H}$, após processos de intercalação, sofre uma transição de fase estrutural o que altera a suas propriedades físicas e químicas. Sendo o processo de intercalação reversível, o sistema pode ser utilizado para a estocagem de energia, desde que na presença de eletrólito não aquoso ou eletrólito sólido.

A fase $2 \mathrm{H}$ após intercalação com lítio e posterior processo de exfoliação em água, forma monolamelas em suspensão, ideais para a preparação de nanocristais para aplicações fotoquímicas e nanocompósitos.

As lamelas podem sofrer um enrolamento durante os processos de síntese, produzindo-se nanotubos e estruturas semelhantes aos fularenos, com potenciais aplicações variadas, semelhantes àquelas propostas para os fulerenos derivados do carbono.

Baseado no descrito acima, conclui-se que apesar da grande aplicação do dissulfeto de molibdênio em vários ramos da indústria, muitas são as potenciais aplicações que ainda deverão ser investigadas no futuro.

\section{AGRADECIMENTOS}

Aos órgãos de fomento do país (CAPES, CNPq e FINEP). Às Instituições, Pesquisadores e Departamentos que colaboraram direta ou indiretamente (LACTEC, LORXI, ETH/Suíça). Prof. Wido H. 
Schreiner pela leitura do manuscrito e sugestões. Aos alunos de iniciação científica, mestrado e doutorado que possibilitaram a obtenção de grande parte dos resultados descritos no artigo.

\section{REFERÊNCIAS}

1. Remmert, P.; Fischer, E.; Hummel, H.; Z. Naturf. 1994, 49b, 1175.

2. Wypych, F.; Schöllhorn, R.; J. Chem. Soc. Chem. Commun. 1992, 1386.

3. Woollam, J.A.; Somoano, R. B.; Mat. Sci. Eng. 1977, 31, 289.

4. Weber, Th.; Muijsers, J. C.; Niemantsverdriet, J. W.; J. Phys. Chem. 1995, 99, 9194.

5. Chianelli, R. R.; Prestridge, E. B.; Pecoraro, T. A.; Deneufville, J. P.; Science 1979, 203, 1105.

6. Remskar, M.; Skraba, Z.; Stadelmann, P.; Levy, F.; Adv. Mat. 2000, 12, 814.

7. Frey, G. L.; Tenne, R.; Matthews, M. J.; Dresselhaus, M. S.; Dresselhaus, G.; J. Mat. Res. 1998, 13, 2412.

8. Jose-Yacaman, M.; Lopez, H.; Santiago, P.; Galvan D. H.; Garzon, I. L.; Reyes, A,; Appl. Phys. Lett. 1996, 69, 1065.

9. Jacobson, A. J.; Chianelli, R. R.; Whitthingham, M. S.; J. Electrochem. Soc. 1979, 2277.

10. Julien, C.; Saikh, S. I.; Nazri, G. A.; ISSI Lett. 1990, 1, 12.

11. Julien, C.; Saikh, S. I.; Nazri, G. A.; Mat. Sci. Eng. 1992, B15, 73.

12. Wilcoxon, J. P.; Newcomer, P. P.; Samara, G. A.; J. Appl. Phys. 1997, 81, 7934.

13. Cohen, S. R.; Rapoport, L.; Ponomarev, E. A.; Cohen, H.; Tsirlina, T.; Tenne, R.; Levy-Clement, C.; Thin Solid Films 1998, 324, 190.

14. Topsoe, H.; Clausen, B. S.; Cat. Rev. Sci. Eng. 1984, 26, 395.

15. Prins, R.; De Beer, V. H. J.; Somorjai, G. A.; Cat. Rev. Sci. Eng. 1989, 31, 1.

16. Furimsky, E.; Cat. Rev. Sci. Eng. 1980, 22, 371.

17. Galiasso, R.; Garcia, W.; Agudelo, M. M. R.; Andreu, P.; Cat. Rev. Sci. Eng. 1984, 26, 445.

18. Song, C.; Saini, A. K.; Yoneyama, Y.; Fuel 2000, 79, 249.

19. Levy, F.; Il Nuovo Cimento 1977, 38, 359.

20. Wang, H. W.; Skeldon, P.; Thompson, G. E.; Wood, G. C.; J. Mat. Sci. 1997, 32, 497.

21. Wang, H. W.; Skeldon, P.; Thompson, G. E.; Wood, G. C.; J. Mat. Sci. 1997, $32,494$.

22. Wang, H. W.; Skeldon, P.; Thompson, G. E; J. Mat. Sci. 1998, 33, 3079

23. Alonso, G.; Del Valle, M.; Cruz, J.; Licea-Claverie, A.; Petranovski, V.; Fuentes, S.; Cat. Lett. 1998, 52, 55.

24. Weber, Th.; Muijsers, J. C.; van Wolput, J. H. M. C.; Verhagen, C. P. J.; Niemantsverdriet, J. W.; J. Phys. Chem. 1996, 100, 14144.

25. Wildervanck, J. C.; Jellinek, F.; Z. Anorg. Allg. Chem. 1964, 328, 309.

26. Mdleleni, M. M.; Hyeon, T.; Suslick, K. S.; J. Am. Chem. Soc. 1998, 120, 6189.

27. Vollath, D.; Szabo, D. V.; Mat. Lett. 1998, 35, 236.

28. Rueda, N.; Bacaud, R.; Vrinat, M.; J. Catal. 1997, 169, 404.

29. Iwata, Y.; Sato, K.; Yoneda, T.; Miki, Y.; Sugimoto, Y.; Nishijima, A., Shimada, H.; Catal. Today 1998, 45, 353.

30. Calais, C.; Matsubayashi, N.; Geantet, C.; Yoshimura, Y.; Shimada, H.; Nishijima, A.; Lacroix, M.; Breysse, M.; J. Catal. 1998, 174, 130.

31. Stevens, G. C.; Edmonds, T.; J. Less Common Met. 1977, 54, 321.

32. Topsoe, H.; Candia, R.; Topsoe, N.; Clausen, B. S.; Bull. Soc. Chim. Belg. 1984, 93, 783.

33. Holl, Y.; Garin, F.; Maire, G.; J. Catal. 1988, 113, 569.

34. Roxlo, C. B.; Daage, M.; Leta, D. P.; Liang, K. S.; Rice, S.; Ruppert, A. F.; Chianelli, R. R.; Solid State Ionics 1986, 22, 97.

35. Louwers, S. P. A.; Prins, R.; J. Catal. 1992, 133, 94.

36. Dungey, K. E.; Curtis, M. D.; Penner-Han, J. E.; J. Catal. 1998, 175, 129

37. Medici, L. A.; Tese de Doutorado, ETH, Zurique, 1995.

38. Wahl, K. J.; Belin, M.; Singer, I. L.; Wear 1998, 214, 212.

39. Imanishi, N.; Toyoda, M.; Takeda, Y.; Yamamoto, O.; Solid State Ionics 1992, 58, 333 .

40. Mulhern, P.; Can. J. Phys. 1989, 67, 1049.
41. Py, M. A.; Haering, R. R.; Can. J. Phys. 1983, 61, 76.

42. Chrissafis, K.; Zamani, M.; Kambas, K.; Stoemenos, J.; Economou, N. A.; Mat. Sci. Eng. B3 1989, 145.

43. Wypych, F.; Seefeld, N.; Denicolo, I.; Quim. Nova 1997, 20, 356.

44. Nakagaki, S.; Mangrich, A. S.; Wypych, F.; Inorg. Chim. Acta 1997, 254, 213

45. Moly-Energy Ltd, 3985 Myrtle Rd, Burnaby, B. C., Canadá.

46. Gonzalez, G.; Santa Ana, M. A.; Benavente, E.; Donoso, J. P.; Bonagamba, T. J.; Melleo, N. C.; Panepucci, H.; Solid State Ionics 1996, 85, 225.

47. Gonzalez, G.; Santa Ana, M. A.; Benavente, E,; Electrochim. Acta 1998 , 43,1327

48. Lemmon, J. P.; Lerner, M. M.; Chem. Mat. 1994, 6, 207.

49. Bissesur, R.; Schindlere, J. J.; Kannewurf, C. R.; Kanatzidis, M.; Mol. Cryst. Liq. Cryst. 1994, 245, 249.

50. Lemmon, J. P.; Wu, J.; Oriakhi, C.; Lerner, M. M.; Electrochim. Acta 1995, $40,2245$.

51. Lara, N.; Ruiz-Hitzki, E.; J. Braz. Chem. Soc. 1996, 7, 193.

52. Wypych, F.; Adad, L. B.; Grothe, M. C.; Quim. Nova 1998, 21, 687.

53. Valeev, E. F.; Zubavitchus, Y. V.; Slovokhotov, Y. L.; Golub, A. S.; Protzenko, G. A.; Novikov, Y. N.; Phys. B. 1995, 208, 569.

54. Kanatzidis, M.; Bissessur, R.; DeGroot, D. C.; Schindler, J. L.; Kannewurf, C. R.; Chem. Mat. 1993, 5, 595.

55. Zubavichus, Y. V.; Slovokhotov, Y. L.; Schilling, P. J.; Tittsworth, R. C.; Golub, A. S.; Protzenko, G.; Novikov, Y. N.; Inorg. Chim. Acta 1998, 280, 211.

56. Golub, A. S.; Shumilova, I. B.; Novikov, Y. N.; Mansot, J. L.; Danot, M.; Solid State Ionics 1996, 91, 307.

57. Bissessur, R.; Heising, J.; Hirpo, W.; Kanatzidis, M.; Chem. Mat. 1996, 8 , 318

58. Heising, J.; Bonhomme, F.; Kanatzidis, M. G.; J. Solid State Chem. 1998, 139,22

59. Gee, M. A.; Frindt, R. F.; Joensen, P.; Morrison, S. R.; Mat. Res. Bull. 1986, $21,543$.

60. Golub, A. S., Shumilova, I. B.; Zubavichus, Y. V.; Jahncke, M.; Suss-Fink, G.; Danot, M.; Novikov, Y. N.; J. Mat. Chem. 1997, 7, 163.

61. Divigalpitiya, W. M. R.; Frindt, R. F.; Morrison, S. R.; Science 1989, 246, 369.

62. Lemaux, S.; Golub, A. S.; Gressier, P.; Ouvrard, G.; J. Solid State Chem. 1999, 147, 336

63. Yang, D.; Westreich, P.; Frindt, R. F.; Nanostruct. Mat. 1999, 12, 467.

64. Golub, A. S., Shumilova, I. B.; Zubavichus, Y. V.; Solovokhotov, Y. L.; Novikov, Y. N.; Marie, A. M.; Danot, M.; Solid State Ionics 1999, 122, 137

65. Heising, J.; Kanatzidis, M.; J. Am. Chem. Soc. 1999, 121, 11720.

66. Wypych, F.; Weber, Th.; Prins, R.; Surf. Sci. 1997, 380, L474.

67. Wypych, F.; Weber, Th.; Prins, R.; Chem. Mat. 1998, 10, 723.

68. Wypych, F.; Solenthaler, C.; Prins, R.; Weber, Th.; J. Solid State Chem. 1999, 144,430 .

69. Wypych, F.; Sollmann, K.; Schoellhorn, R.; Mat. Res. Bull. 1992, 27, 545.

70. Wypych, F.; Tsunoda, M.; Quim. Nova 1993, 16, 543.

71. Wypych, F.; Tsunoda, M.; Quim. Nova 1994, 17, 9.

72. Wypych, F.; Schoellhorn, R,; Quim. Nova 1996, 19, 5.

73. Wypych, F,; Tese de Doutorado, PUC/RJ, 1992.

74. Heising, J.; Kanatzidis, M. G.; J. Am. Chem. Soc. 1999, 121, 638.

75. Dungey, K. E.; Curtis, M. D.; Penner-Hahn, J. E.; Chem. Mat. 1998, 10, 2152.

76. Lerf, A.; Schoellhorn, R.; Inorg. Chem. 1977, 16, 2950.

77. Gourmelon, E.; Lignier, O.; Hadonda, H.; Couturier, G.; Bernede, J. C.; Tedd, J.; Pouzel, J.; Salardenne, J., Solar Energy Mat. Solar Cells 1997, 46,115 .

78. Gourmelon, E.; Pouzet, J.; Bernede, J. C.; Hadouda, H.; Khelil, A.; Leny, R.; Mat. Chem. Phys. 1999, 58, 280.

79. Thurston, T. R.; Wilcoxon, J. P.; J. Phys. Chem. B. 1999, 103, 11.

80. Gouvea, C. A. K.; Wypych, F.; Moraes, S. G.; Duran, N.; Nagata, N.; Peralta-Zamora P.; Chemosphere 2000, 40, 433.

81. Gouvea, C. A. K.; Wypych, F.; Moraes, S. G.; Duran, N.; Peralta-Zamora P.; Chemosphere 2000, 40, 427.

82. Scholz, G. A.; Morrison, S. R.; Can. J. Chem. 1989, 67, 862. 